\title{
Revealing the extended radio emission from the gamma-ray binary HESS J0632+057
}

\author{
J. Moldón, M. Ribó, and J. M. Paredes \\ Departament d'Astronomia i Meteorologia, Institut de Ciències del Cosmos (ICC), Universitat de Barcelona (IEEC-UB), \\ Martí i Franquès 1, 08028 Barcelona, Spain \\ e-mail: [jmoldon;mribo; jmparedes]@ub.edu
}

Received 25 July 2011 / Accepted 12 August 2011

\begin{abstract}
Context. After the detection of a 321-day periodicity in X-rays, HESS J0632+057 can be robustly considered a new member of the selected group of gamma-ray binaries. These sources are known to show extended radio structure on scales of milliarcseconds (mas). Aims. We present the expected extended radio emission on mas scales from HESS J0632+057.

Methods. We observed HESS J0632+057 with the European VLBI Network (EVN) at $1.6 \mathrm{GHz}$ in two epochs: during the January/February $2011 \mathrm{X}$-ray outburst and 30 days later.

Results. The VLBI image obtained during the outburst shows a compact $\sim 0.4 \mathrm{mJy}$ radio source, whereas 30 days later the source has faded and appears extended, with a projected size of $\sim 75 \mathrm{AU}$. The peak of the emission is displaced between runs $21 \pm 5 \mathrm{AU}$, which is bigger than the orbit size. The position of the radio source is compatible with the Be star MWC 148, which sets the proper motion

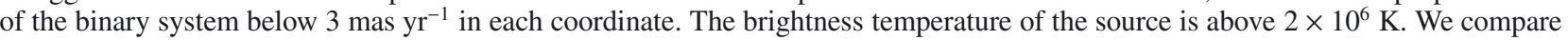
the multiwavelength properties of HESS J0632+057 with those of the previously known gamma-ray binaries.

Conclusions. HESS J0632+057 displays extended and variable non-thermal radio emission. Its morphology, size, and displacement on AU scales are similar to those found in the other gamma-ray binaries, PSR B1259-63, LS 5039, and LS I +61 303, supporting a similar nature for HESS J0632+057.
\end{abstract}

Key words. radio continuum: stars - X-rays: binaries - stars: emission-line, Be - gamma rays: stars - stars: individual: MWC148

\section{Introduction}

Gamma-ray binaries are binary systems containing a compact object orbiting a companion star, which are able to produce $\mathrm{GeV}$ and/or TeV emission and show the peak of the spectral energy distribution (SED) at $\mathrm{MeV}-\mathrm{GeV}$ energies. The three "classical" gamma-ray binaries are PSR B1259-63, LS 5039, and LS I +61 303, which have been unambiguously detected as point-like sources at $\mathrm{TeV}$ energies (see the reviews in Paredes 2008; Bosch-Ramon \& Barkov 2011). The broadband emission from radio to very high energy (VHE) gamma rays of these sources is variable and periodic, following the orbital period of the binary system. These three sources present variable milliarcsecond (mas) scale radio structures that have been detected with Very Long Baseline Interferometry (VLBI). We note that other binary systems have been detected up to $\mathrm{GeV}$ energies, but not confirmed at TeV energies (e.g., Tavani et al. 2009; Abdo et al. 2009, for Cygnus X-3), including the gamma-ray binary candidate 1FGL J1018.6-5856 (Corbet et al. 2011; Pavlov et al. 2011). Two main scenarios have been proposed to understand the multiwavelength behaviour of gamma-ray binaries, the basic difference being the nature of the particle accelerator. In one of them, particles are accelerated in the jets of a microquasar (see, e.g., Bosch-Ramon \& Khangulyan 2009). In the other one, particles are accelerated in the shock between the relativistic wind of a young non-accreting pulsar and the stellar wind of the massive companion star (see Tavani \& Arons 1997; Dubus 2006).

HESS J0632+057 is the most recent addition to the selected group of gamma-ray binaries. The H.E.S.S. Collaboration found the point-like TeV source HESS J0632+057 (Aharonian et al. 2007), which was variable at $\mathrm{TeV}$ energies (Acciari et al. 2009). The source has a variable $X$-ray counterpart (Hinton et al. 2009; Acciari et al. 2009), as well as a variable radio counterpart (Skilton et al. 2009). The massive B0pe star MWC 148 (HD 259440), located at $\sim 1.5 \mathrm{kpc}$, was proposed as the optical counterpart (Hinton et al. 2009). The SED from radio to TeV energies is very similar to the one of LS I +61303 , which also contains a Be star (Hinton et al. 2009). All these results suggest that HESS J0632+057 is a new gamma-ray binary, displaying an SED one order of magnitude fainter than LS I +61 303. This is an important fact that could shed light on the luminosity distribution of this new population of binary systems in the Galaxy.

Recently, a periodicity of $321 \pm 5 \mathrm{~d}$ has been revealed thanks to long-term X-ray observations conducted with Swift/XRT, strongly supporting the binary nature of the source (Bongiorno et al. 2011). This orbital period is between the period of PSR B1259-63 (1237 days) and the one of LS I +61 303 (26.5 days). The X-ray light curve, which covers three orbital cycles, shows a bright peak and a fainter secondary peak separated $\sim 0.5$ in phase. On the other hand, optical spectroscopic observations have not allowed the binary nature of the source to be unveiled up to now (Aragona et al. 2010; Casares et al. 2011).

The last bright X-ray outburst of HESS J0632+057 occurred in February 2011. Falcone et al. (2011) report increased X-ray activity detected by Swift/XRT between January 23 and February 6, 2011 (MJD 55 584-55 598). The VERITAS Collaboration reports increased activity at energies above $300 \mathrm{GeV}$ between February 7 and 8, 2011 (MJD 55 599-55 600) 
(Ong 2011). The MAGIC Collaboration reported increased gamma-ray flux above $200 \mathrm{GeV}$ during February 7-9, 2011 (MJD 55 599-55 601) (Mariotti 2011), confirming the VHE active state and lowering the measured energy threshold. During the outburst, we observed the source with the European VLBI Network (EVN) to explore its radio emission on mas scales.

The non-thermal radio emission produced by gamma-ray binaries has been observed on scales of 1-100 mas. Massi et al. (2004) report a jet-like and precessing structure with an extension of 10-80 mas (25-200 AU) from LS I +61 303. VLBA observations at $8 \mathrm{GHz}$ along the orbital cycle (Dhawan et al. 2006) have shown that the $\sim 7$ mas (14 AU) structure follows a cometary behaviour, while the peak of the emission traces approximately an ellipse. For LS 5039, extended radio emission has been found at $5 \mathrm{GHz}$, with projected sizes of 6 mas (15 AU) with the VLBA (Paredes et al. 2000; Ribó et al. 2008), and between 60 and 300 mas (150-750 AU) with the EVN and MERLIN (Paredes et al. 2002). Observations with the VLBA covering an orbital cycle show periodic morphological variability and hints of peak displacement (Moldón et al. 2011c). Recently, Moldón et al. (2011a) has shown that PSR B1259-63, the only gamma-ray binary that is known to contain a pulsar, displays emission with a projected extension of 50 mas (120 AU) at $2.3 \mathrm{GHz}$ during periastron. The peak of the radio nebula is detected at distances between 10 and 50 AU from the system.

Here we present the first VLBI radio images of HESS J0632+057, obtained with the EVN during and after the high-energy activity period in early 2011 (preliminary results were presented in Moldón et al. 2011b). The observations and data reduction are described in Sect. 2. The results can be found in Sect. 3. A discussion of these results in the context of gamma-ray binaries can be found in Sect. 4 .

\section{Observations and data reduction}

Following the report of X-ray activity from HESS J0632+057 between January 23 and February 6, 2011 (Falcone et al. 2011), we observed the source with the EVN in target of opportunity (ToO) mode in two epochs separated by 30 days. The first radio continuum observation (run A) was conducted at $1.6 \mathrm{GHz}(18 \mathrm{~cm})$ during eight hours on February 15, 2011 (MJD 55607), UTC 15:50 to 00:00. The antenna network consisted in seven antennas: Effelsberg, Jodrell Bank Lovell, Medicina, Onsala, Torun, Westerbork, and Hartebeesthoek, providing baselines ranging from 200 to $8000 \mathrm{~km}(200$ to $1000 \mathrm{~km}$ without Hartebeesthoek). A data rate of $1024 \mathrm{Mbps}$ per station was directly streamed to the central processor at JIVE and correlated in real time (e-VLBI). The rapid response of the e-EVN observations and correlation allowed us to perform a second ToO (run B) in a disk-recorded session with 12 EVN antennas: Effelsberg, Jodrell Bank Lovell, Medicina, Onsala, Torun, Westerbork, Hartebeesthoek, Svetloe, Badary, Zelenchukskaya, Nanshan (Urumqi), and Sheshan (Shanghai), providing baselines ranging from 200 to $10000 \mathrm{~km}$. The observation lasted ten hours and was conducted on March 17, 2011 (MJD 55 637), UTC 13:00 to 23:00, with a data rate of 1024 Mbps. This second epoch provides considerably better sensitivity. The EVN project codes for the observations are RR005 and RM006, respectively.

Both observations have a similar structure, switching between HESS J0632+057 and the phase reference calibrator J0619+0736, separated 3.9, with cycling times of five minutes to avoid losing phase coherence. The phase reference calibrator was correlated at the position $\alpha_{\mathrm{J} 2000.0}=06^{\mathrm{h}} 19^{\mathrm{m}} 09^{\mathrm{s}} .9710$ and $\delta_{\mathrm{J} 2000.0}=07^{\circ} 36^{\prime} 41^{\prime \prime} .220$ in the frame of ICRF (Goddard VLBI global solution $2010 \mathrm{a}^{1}$ ). This position has absolute uncertainties of 2.0 and 1.1 mas in right ascension and declination, respectively. The fringe finders DA193, 3C147, and OQ208 were observed during run A and 0528+134, and DA193 during run B.

The data reduction was performed in AIPS $^{2}$. Flagging based on predicted off-source times, owing to slewing or failures, was applied using UVFLG. A priori visibility amplitude calibration used the antenna gains and the system temperatures measured at each station. The amplitude calibration was improved by scaling the individual antenna gains by a factor obtained from the phase calibrator and fringe-finder models. We used ionospheric total electron content (TEC) models based on GPS data obtained from the CDDIS data archive $^{3}$ to correct the global phase variations due to the ionosphere. The parallactic angle correction was applied with VLBAPANG. Fringe fitting on the phase reference calibrator was performed with the AIPS task FRING, and the solutions were applied to the target source. The instrumental bandpasses were corrected using BPASS. The data were averaged in frequency and time, and clean images were produced with IMAGR. A cell size of 1 mas was used for cleaning both epochs. The images were produced using a weighting scheme with robust parameter 0 for run $A$, and 2 (which is slightly more sensitive to extended emission) for run B. A tapering of $30 \mathrm{M} \lambda$ was applied to avoid the presence of possible unreliable highresolution features due to sidelobes of the synthesized beam. No self-calibration of the data was possible because of the low flux density of the target source.

\section{Results}

The resulting VLBI images at $1.6 \mathrm{GHz}$ are shown in Fig. 1. Run A shows a $410 \pm 90 \mu \mathrm{Jy}$ point-like source. Run B, observed 30 days later, shows a $180 \pm 30 \mu \mathrm{Jy}$ (measured with TVSTAT within AIPS) faint source with extended emission towards north. The $1 \sigma \mathrm{rms}$ noise close to the source is $50 \mu \mathrm{Jy}$ beam $^{-1}$ and $13 \mu \mathrm{Jy}$ beam $^{-1}$, respectively. We note that the flux density of the extended emission detected in run B is below the rms noise limit of the image of run A. In Table 1 we show some parameters of the observations, the obtained synthesized beam, and the fitted components to the source (Gaussian components obtained with JMFIT within AIPS).

The emission in run A is described by a component located at $\alpha_{\mathrm{J} 2000.0}=06^{\mathrm{h}} 32^{\mathrm{m}} 59^{\mathrm{s}} .2567(1)$ and $\delta_{\mathrm{J} 2000.0}=05^{\circ} 48^{\prime} 01^{\prime \prime} .162(2)$. The values in parenthesis refer to the uncertainty in the last digit (1.3 and 2 mas, respectively), and correspond to the formal errors of the fit. Run B is described well by a point-like core (see Table 1) and an extended component (which has an intrinsic PA of $4 \pm 20^{\circ}$ ) located at $21 \pm 6$ mas in $\mathrm{PA} 30 \pm 10^{\circ}$ with respect to Core2. The total extension of the source is $\sim 50$ mas.

The relative astrometry shows that the peak of the emission suffers a total displacement of $14 \pm 3$ mas in PA $190 \pm 9^{\circ}$ between runs $\mathrm{A}$ and $\mathrm{B}$. This displacement is aligned with the direction of the extended emission. The separation between the peak positions corresponds to a projected linear distance of $21 \pm 5 \mathrm{AU}$. Covering this distance in the 30 days between runs requires a constant velocity of $1200 \pm 300 \mathrm{~km} \mathrm{~s}^{-1}$.

\footnotetext{
${ }^{1}$ http://gemini.gsfc.nasa.gov/solutions/2010a/2010a. html

2 The NRAO Astronomical Image Processing System. http://www . aips.nrao. edu/

3 The Crustal Dynamics Data Information System. http://cddis.nasa.gov/
} 
J. Moldón et al.: Extended radio emission of HESS J0632+057
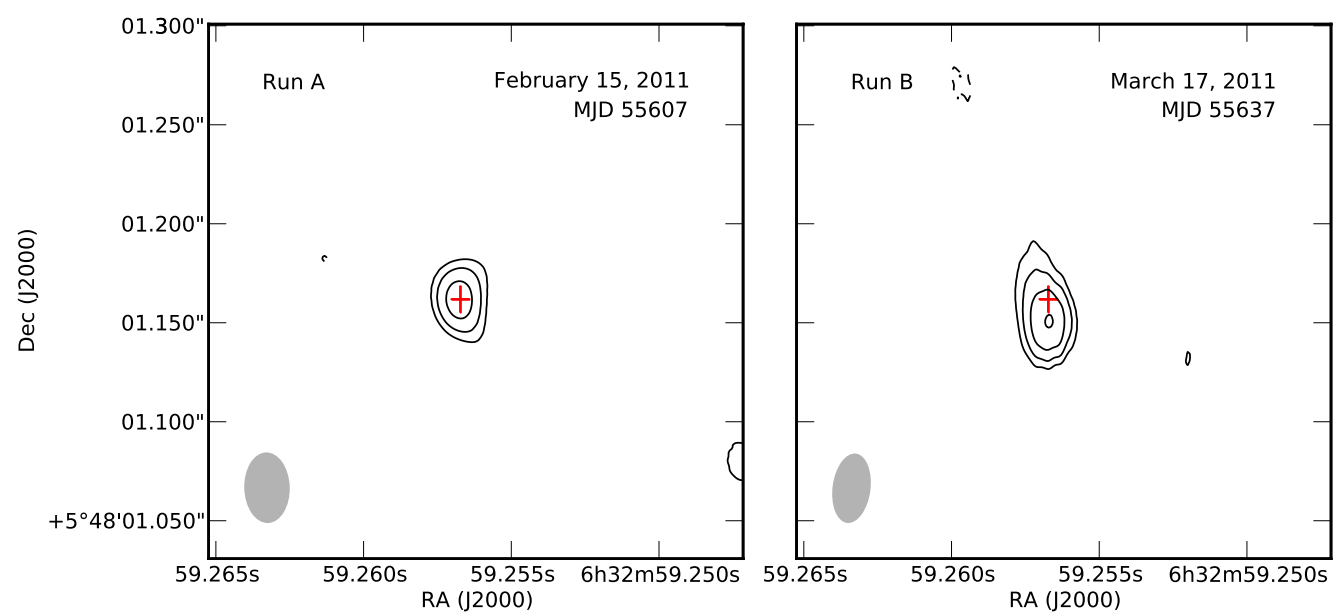

Fig. 1. EVN radio images of HESS J0632+057 at $1.6 \mathrm{GHz}$ during the X-ray outburst and 30 days later. North is up and east to the left. The observation dates are quoted at the top of each panel. The synthesized beam (see Table 1) is displayed in the bottom-left corner of each image. The red crosses mark the position and $3 \sigma$ uncertainty of the fitted component of run A. For each image, the displayed contours start at $3 \sigma$ and increase by factors of $2^{1 / 2}$; the $1 \sigma \mathrm{rms}$ close to the source is $50 \mu \mathrm{Jy}_{\text {beam }}^{-1}$ in run A and $13 \mu \mathrm{Jy}_{\text {beam }}{ }^{-1}$ in run B.

Table 1. Observation parameters and Gaussian components fitted to the images.

\begin{tabular}{|c|c|c|c|c|c|c|c|c|c|c|c|c|}
\hline Run & MJD & $\begin{array}{l}\text { Num. } \\
\text { anten. }\end{array}$ & $\begin{array}{c}\theta_{\mathrm{HPBW}} \\
{\left[\mathrm{mas}^{2}\right]}\end{array}$ & $\begin{array}{c}\theta_{\mathrm{PA}} \\
{\left[{ }^{\circ}\right]}\end{array}$ & Comp. & $\begin{array}{c}S_{\text {peak }} \\
{\left[\mu \mathrm{Jy} \mathrm{b}^{-1}\right]}\end{array}$ & $\begin{array}{c}S_{1.6 \mathrm{GHz}} \\
{[\mu \mathrm{Jy}]}\end{array}$ & $\begin{array}{l}\Delta \alpha^{a} \\
{[\mathrm{mas}]}\end{array}$ & $\begin{array}{l}\Delta \delta^{a} \\
{[\mathrm{mas}]}\end{array}$ & $\begin{array}{c}\text { Maj. axis } \\
\text { [mas] }\end{array}$ & $\begin{array}{c}\text { Min. axis } \\
\text { [mas] }\end{array}$ & Type \\
\hline $\mathrm{A}$ & 55607.83 & 7 & $36 \times 23$ & 2 & Core1 & $340 \pm 50$ & $410 \pm 90$ & - & - & $12 \pm 13$ & $12 \pm 10$ & Point-like \\
\hline B & 55637.75 & 12 & $35 \times 19$ & -7 & $\begin{array}{l}\text { Core2 } \\
\text { Extended }\end{array}$ & $\begin{array}{l}81 \pm 14 \\
56 \pm 13\end{array}$ & $\begin{array}{r}90 \pm 25 \\
110 \pm 40\end{array}$ & $\begin{array}{r}-3 \pm 2 \\
6 \pm 3\end{array}$ & $\begin{array}{r}-14 \pm 3 \\
5 \pm 6\end{array}$ & $\begin{array}{r}9 \pm 11 \\
48 \pm 18\end{array}$ & $\begin{array}{l}12 \pm 12 \\
11 \pm 10\end{array}$ & $\begin{array}{l}\text { Point-like } \\
\text { Extended }\end{array}$ \\
\hline
\end{tabular}

Notes. ${ }^{(a)}$ Displacements with respect to the red crosses in Fig. 1.

\section{Discussion}

The first VLBI image of HESS J0632+057 at $1.6 \mathrm{GHz}$, taken a few days after the peak of the January 2011 X-ray outburst, shows a compact $410 \mu \mathrm{Jy}$ point-like source (see Fig. 1). After 30 days, the source displays one-sided extended emission with a total flux density of $180 \mu \mathrm{Jy}$ and with an extension of 50 mas (75 AU assuming a distance to the system of $1.5 \mathrm{kpc}$ ) in PA $30^{\circ}$. The peak of the emission is displaced 14 mas (21 AU) in PA $190^{\circ}$. We note that the rms noise of the first image is above the flux density of the extended emission in the second image.

Even though the orbital parameters of HESS J0632+057 are unknown, the size of the orbit can be derived from the orbital period (321 days), assuming a mass of the compact object of 1.4 $M_{\odot}$, and a Be star of $16 \pm 3 M_{\odot}$ (Aragona et al. 2010). The semimajor axis of the orbit is $2.4 \pm 0.1 \mathrm{AU}$, which corresponds to a projected distance of $1.6 \pm 0.1$ mas (or less considering the inclination and the argument of the periastron of the orbit). The peak displacement reported here, 14 mas, cannot be explained by any motion within the orbit.

The measured position in run A is clearly compatible with the UCAC3 catalogue position of the Be star MWC 148, which has an uncertainty of 14 mas in each coordinate (Zacharias et al. 2010), therefore the detected radio source is unambiguously related to the Be star. We computed the proper motion of the source using the position from run A with an uncertainty of 25 mas to include possible peak displacements, the position of MWC 148 from UCAC3, obtained at mean epoch 1984.2 (Zacharias et al. 2010), and the VLA-D and GMRT positions from Skilton et al. (2009) (with uncertainties of 300 and 500 mas, respectively). Despite the time span of 27 years of observations, the proper motion is compatible with no motion. The result is $\mu_{\alpha} \cos (\delta)=$

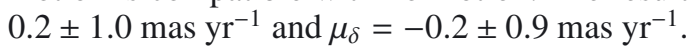

The brightness temperature corresponding to the radio source in run A (see Table 1) at $1.6 \mathrm{GHz}$ is $2 \times 10^{6} \mathrm{~K}$. Such a high value, together with non-simultaneous radio observations suggesting a negative spectral index around $\sim-0.6$ (Skilton et al. 2009), rules out a thermal emission mechanism. Non-thermal synchrotron radiation remains, therefore, as the most plausible interpretation for the HESS J0632+057 radio emission. In this case, and assuming equipartition between the relativistic electrons and the magnetic field, the observed properties correspond to a total energy between 0.1 and $100 \mathrm{GHz}$ of $4 \times 10^{38} \mathrm{erg}$, with an equipartition magnetic field of $\sim 0.02 \mathrm{G}$. These values are approximately ten times lower than those obtained for LS 5039 from VLBI observations (Paredes et al. 2000).

The gamma-ray binaries PSR B1259-63, LS 5039, and LS I +61 303 show a radio morphology with a central core and one-sided extended radio emission on scales of a few AU (although bipolar extended emission has also been detected at some orbital phases: Dhawan et al. 2006; Ribó et al. 2008; Moldón et al. 2011a,c). In all these cases, the authors find morphological changes on timescales close to the orbital period, and displacements of the peak of the emission bigger than the orbit size. The morphology and peak displacement reported here show that the radio emission of HESS J0632+057 is similar to the ones observed in the other known gamma-ray binaries, suggesting a similar nature for HESS J0632+057.

In Fig. 2 we show the phase-folded light curve of the X-ray data from Bongiorno et al. (2011), together with radio (top panel) and TeV (bottom panel) data. Our two EVN measurements suggest a radio flux decrease of the most compact region 


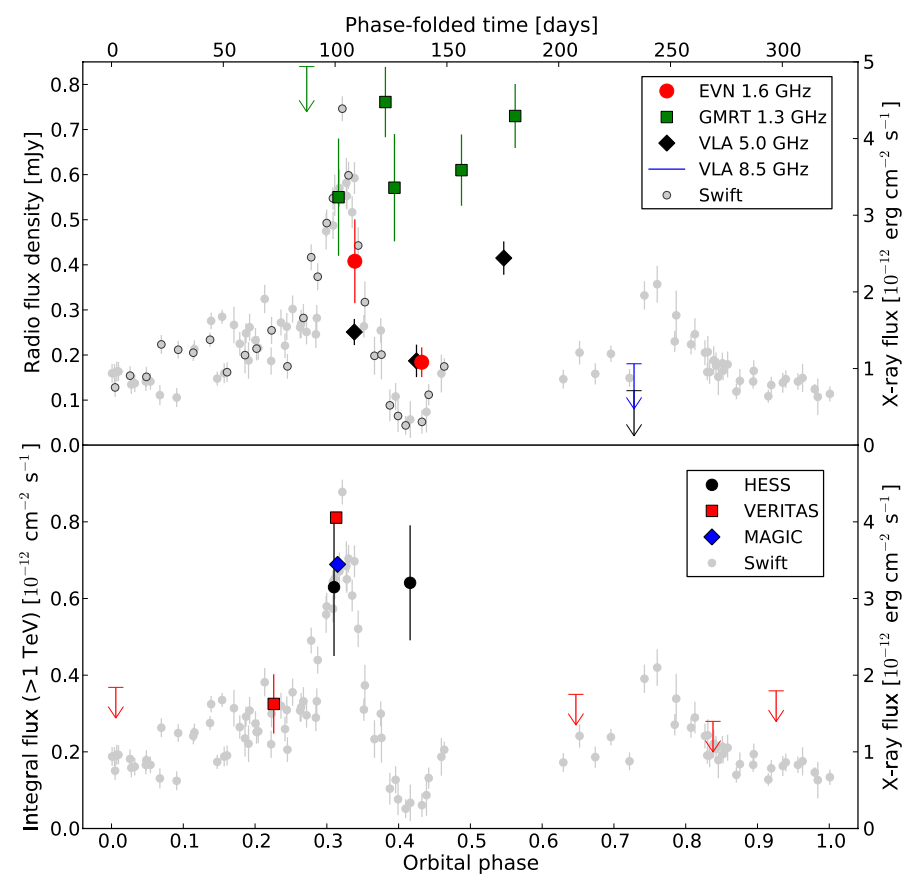

Fig. 2. Multiwavelength light curve of HESS J0632+057. The data were folded with a period of 321 days. Zero phase has been arbitrarily defined at MJD 54857 . The grey circles are X-ray observations by Swift/XRT (0.3-10 keV) from Bongiorno et al. (2011). Top: the red circles are the EVN observations from this paper. The other radio data are from Skilton et al. (2009). The black circles (filled in grey) are Swift/XRT data obtained during the same cycle as the EVN data. Bottom: TeV emission from Acciari et al. (2009); Ong (2011); Mariotti (2011); Mukherjee (2011).

of the source similar to the X-ray one after the outburst, although at a slower rate. However, a VLBI monitoring of the outburst is required to understand the flux changes better. On the other hand, a persistent and higher flux was measured in previous lower resolution GMRT observations at a similar frequency up to $\sim 100$ days after the outburst. This can be explained by the presence of diffuse (resolved) emission, which is lost on VLBI scales (the shortest significant baseline in our data samples spacial frequencies around $1 \mathrm{M} \lambda$, which corresponds to angular scales of 200 mas).

LS I +61 303 shows an X-ray outburst before apastron that is correlated with the TeV outburst (Anderhub et al. 2009; Zabalza et al. 2011). The duration of the X-ray outburst of HESS J0632+057 is $\sim 5$ times longer (although $\sim 0.1$ in phase in both cases). We computed non-simultaneous TeV/X-ray flux ratios during the 2011 outburst (around phase 0.31) and compared them with the ones of LS I +61 303. The TeV/X-ray flux ratio of HESS J0632+057 is $\sim 5$ higher (the X-ray flux is lower, or the $\mathrm{TeV}$ emission is higher) than the one of LS I +61303 . This is consistent with a lower magnetic field in HESS J0632+057. On the other hand, $\mathrm{TeV}$ emission has been detected at similar levels during the X-ray dip, and thus does not follow the X-ray light curve (Fig. 2). When comparing the average radio $(0.7 \mathrm{mJy})$ to $\mathrm{X}$-ray flux ratios, we see that the X-ray flux is higher, or the radio emission is lower, in HESS J0632+057 by a factor $\sim 30$. The outbursts from these two sources are observationally very different, although the lack of information regarding orbital parameters prevents any further discussion.

As a final comment, we could interpret the displacement between the peaks of runs A and B (14 mas in 30 days) as produced by the proper motion of a blob ejected within a microquasar (see
Mirabel \& Rodríguez 1994 for GRS 1915+105). A blob velocity above $0.1 \mathrm{c}$ requires that the jet is pointing towards the observer with an angle below $2^{\circ}$, which would be either very restrictive or fine tuned.

In conclusion, HESS J0632+057 displays extended and variable radio emission at 50-100 AU scales, with a projected displacement of the peak of the emission of $21 \mathrm{AU}$ in 30 days. Similar morphologies and behaviours have been found in the other gamma-ray binaries. However, a more detailed monitoring of the variability of the source with high-sensitivity VLBI observations along the orbital cycle is required to measure the total extension and morphology of the extended emission, to measure the morphologic and astrometric changes at different orbital phases, and to search for periodicity in these changes.

Acknowledgements. We thank the EVN PC Chair, Tiziana Venturi, for supporting our ToO observations and to the EVN stations who made this possible. e-VLBI developments in Europe are supported by NEXPReS, an Integrated Infrastructure Initiative (I3), funded by the European Union Seventh Framework Programme (FP7/2007-2013) under grant agreement RI-261525. The European VLBI Network (http://www.evlbi.org/) is a joint facility of European, Chinese, South African, and other radio astronomy institutes funded by their national research councils. We acknowledge support by the Spanish Ministerio de Ciencia e Innovación (MICINN) under grants AYA2010-21782-C03-01 and FPA2010-22056-C06-02. J.M. acknowledges support by MICINN under grant BES-2008-004564. M.R. acknowledges financial support from MICINN and European Social Funds through a Ramón y Cajal fellowship. J.M.P. acknowledges financial support from ICREA Academia.

\section{References}

Abdo, A. A., Ackermann, M., Ajello, M., et al. 2009, Science, 326, 1512 Acciari, V. A., Aliu, E., Arlen, T., et al. 2009, ApJ, 698, L94

Aharonian, F. A., Akhperjanian, A. G., Bazer-Bachi, A. R., et al. 2007, A\&A, 469, L1

Anderhub, H., Antonelli, L. A., Antoranz, P., et al. 2009, ApJ, 706, L27 Aragona, C., McSwain, M. V., \& De Becker, M. 2010, ApJ, 724, 306 Bongiorno, S. D., Falcone, A. D., Stroh, M., et al. 2011, ApJ, 737, L11 Bosch-Ramon, V., \& Barkov, M. V. 2011, A\&A, submitted [arXiv: 1105.6236] Bosch-Ramon, V., \& Khangulyan, D. 2009, IJMPD, 18, 347

Casares, J., Ribo, M., Paredes, J. M., et al. 2011, ATel, \#3209

Corbet, R. H. D., Cheung, C. C., Kerr, M., et al. 2011, ATel, \#3221

Dhawan, V., Mioduszewski, A., \& Rupen, M. 2006, in Proc. VI Microquasar Workshop: Microquasars and Beyond, ed. T. Belloni, 52.1

Dubus, G. 2006, A\&A, 456, 801

Falcone, A., Bongiorno, S., Stroh, M., \& Holder, J. 2011, ATel, \#3152

Hinton, J. A., Skilton, J. L., Funk, S., et al. 2009, ApJ, 690, L101

Mariotti, M. 2011, ATel, \#3161

Massi, M., Ribó, M., Paredes, J. M., et al. 2004, A\&A, 414, L1

Mirabel, I. F., \& Rodríguez, L. F. 1994, Nature, 371, 46

Moldón, J., Johnston, S., Ribó, M., Paredes, J. M., \& Deller, A. T. 2011a, ApJ, 732, L10

Moldón, J., Ribó, M., \& Paredes, J. M. 2011b, ATel, \#3180

Moldón, J., Ribó, M., \& Paredes, J. M. 2011c, in High-Energy Emission from Pulsars and their Systems, ed. D. F. Torres, \& N. Rea, Astrophys. Space Sci. Proc. (Berlin, Heidelberg: Springer-Verlag), 1

Mukherjee, R. 2011, in High Energy Phenomena in Relativistic Outflows III, ed. J. M. Paredes, M. Ribó, F. A. Aharonian, \& G. E. Romero

Ong, R. A. 2011, ATel, \#3153

Paredes, J. M. 2008, in AIP Conf. Ser. 1085, ed. F. A. Aharonian, W. Hofmann, \& F. Rieger, 157

Paredes, J. M., Martí, J., Ribó, M., \& Massi, M. 2000, Science, 288, 2340

Paredes, J. M., Ribó, M., Ros, E., Martí, J., \& Massi, M. 2002, A\&A, 393, L99

Pavlov, G. G., Misanovic, Z., Kargaltsev, O., \& Garmire, G. P. 2011, ATel, \#3228

Ribó, M., Paredes, J. M., Moldón, J., Martí, J., \& Massi, M. 2008, A\&A, 481, 17

Skilton, J. L., Pandey-Pommier, M., Hinton, J. A., et al. 2009, MNRAS, 399, 317

Tavani, M., \& Arons, J. 1997, ApJ, 477, 439

Tavani, M., Bulgarelli, A., Piano, G., et al. 2009, Nature, 462, 620

Zabalza, V., Paredes, J. M., \& Bosch-Ramon, V. 2011, A\&A, 527, A9

Zacharias, N., Finch, C., Girard, T., et al. 2010, AJ, 139, 2184 